\title{
Swarming ring patterns in bacterial colonies exposed to ultraviolet radiation
}

\author{
Anna M. Delprato ${ }^{1,2}$, Azadeh Samadani ${ }^{1}$, A. Kudrolli ${ }^{1}$, , and L.S. Tsimring ${ }^{3}$ \\ 1 Department of Physics, Clark University, Worcester, MA 01610 \\ 2 Department of Biology, Clark University, Worcester, MA 01610 \\ ${ }^{3}$ Institute for Nonlinear Science, University of California, San Diego, CA 92093-0402
}

(November 3, 2018)

\begin{abstract}
We report a novel morphological transition in a Bacillus subtilis colony initially growing under ambient conditions, after ultraviolet radiation exposure. The bacteria in the central regions of the colonies are observed to migrate towards the colony edge forming a ring during uniform spatial exposure. When the radiation is switched off, the colonies were observed to grow both inward into the evacuated regions as well as outward indicating that the pattern is not formed due to depletion of nutrients at the center of the colony. We also propose a reaction-diffusion model in which wastelimited chemotaxis initiated by the UV radiation leads to the observed phenomenology.

PACS numbers: 87.18.Hf, 47.20.Hw, 87.50.Gi, 87.23.Cc
\end{abstract}

Bacterial colony growth on semi-solid agar has served as a model system to understand self-organization of microscopic biological elements to form complex patterns [1] 3]. Although interaction between individuals may be well known, it is difficult to determine how complex patterns form for example due to chemotaxis. Random walk and reaction diffusion models have been also developed to find the minimal mechanisms that lead to the formation of the observed patterns [3 0 ]. In most of these studies, changes in the environment occur due to the slow depletion of nutrients and excretion of waste.

The modeling of the response of living organisms to a change in environment is an important issue of current interest. An example is the effect of ultraviolet (UV) radiation on biological systems prompted by stratospheric ozone depletion. Recent studies have modeled the response to externally imposed change on bacterial colonies using the Fisher wave equation with added convection terms [8 [9]. Experiments using ultraviolet illumination pattern changes on Bacillus subtilis colonies have shown the relevance of such modeling [10]. However, a delay in the response of the bacterial colony to changes in the environment was also noted. These studies raise interesting questions on how to model biological systems that may undergo growth mode changes and show history dependence in response to environmental changes.

In this paper, we report the spatio-temporal response of colonies of Bacillus Subtilis growing on nutrient rich agar to a sudden change in the environment. Bacillus Subtilis is a $9 \mu \mathrm{m} \times 2 \mu \mathrm{m}$ motile bacterium that can be often found in soil. The change is hostile and is implemented by exposing the colony to uniform UV radiation. The colony which grows uniformly before exposure to UV radiation, is not observed to simply stop due to the radiation but undergo a morphology change. Most of the bacteria are observed to migrate to the edge of the colony even though this does not help the bacteria to evade the radiation. To systematically study the spatio- temporal response of the colony, we measured the change in the bacterial density as a function of the strength of the UV radiation for the wild type and DNA repair mutants, uvrA and uvrA recA of the bacterium. We also propose a simple reaction-diffusion model which reproduces most of the observed phenomenology. The model operates with three densities: bacteria, waste, and chemoattractant, and describes a waste-limited chemotaxis initiated by the UV radiation. The bacteria migrate towards the edges because the waste concentration is reduced at the edges of the colony.

Experiments were performed in $15 \mathrm{~cm}$ diameter Plexiglas petri dishes containing a thin layer of nutrient agar (7 grams/liter of bacto peptone and 3 grams/liter agar). Under these conditions the growth of the bacterial colony can be approximately modeled by the Fisher wave equation 11,10. The bacterial colony was initiated with an inoculating needle at a single point source at the center of the petri dish and the bacteria grew in a thin layer at the surface of the medium. The colony is first allowed to grow in the absence of UV radiation. The initial adaptation time and subsequent growth rate of the colony depends on the temperature and for most of the experiments a temperature of $24^{\circ} \mathrm{C}$ was used. At this temperature, the colony front was observed to grow slowly for the first 26 hours and then increased reaching a constant front speed $v_{f}=0.3 \mu \mathrm{m} / \mathrm{s}$. The colony was then exposed to uniform UV radiation over a time interval $t_{u v}$ using a fluorescent bulb, (Philips F15T8) which radiates in the range of 310-400 nm (UV-A and UV-B). An example of the spatio-temporal response in a colony of Bacillus subtilis, uvrA under UV radiation is shown in Fig. 1](a)-(c).

We note that the circular symmetry of the patterns is a consequence of the point inoculation and the subsequent disk shaped colony which develops before the colony is exposed to UV radiation. If the bacteria is grown in a ribbon shape using a line inoculation pattern, they still migrate to the edges. For simplicity, we performed quan- 
titative experiments with colonies using point inoculation.

To further investigate the cause of the migration of the bacterial population, we observed the recovery of the colony after exposure to radiation. Images of the recovering colony are shown in Fig. 11(d)-(f) and it can be noted that the colony recovers almost uniformly in both directions. The colony grows outward to fresh regions and also inward into the evacuated central regions.

The radius of the circular colony before exposure, during exposure and after exposure was plotted as a function of time in Fig. 2. We observed that the growth of the front of the colony during exposure is negligible compared to the growth before exposure. After radiation is switched off, it can be observed that cell division resumes following a "recovery" time $t_{r}$, which increases with the strength of the radiation. Although, the front speed on the outside edge is greater than the inside edge, clearly the central regions of the colony are hospitable. Therefore, the initial evacuation of the colony at the center is not due to depletion of nutrients. Thus the formation of the swarm ring like patterns has clearly a different mechanism than observed previously [12,7].

To quantitatively show the rearrangement of the bacterial colony as a function of time, the bacterial density $n(r)$ as a function of distance $r$ from the center is plotted in Fig. 3(a). The density of the bacteria scales linearly with light intensity and therefore the density distribution of the colony is obtained from the images using a CCD camera. At approximately 24 hours the density of the bacteria is considerably higher at the edge of the colony compared to the colony center. After 70 hours the bacteria almost completely evacuate the center of the colony.

In Fig. 3(b), we show the dependence of the strength of the UV radiation $I$ on the colony. The cross section of the colony was plotted after the colonies were allowed to grow and were exposed to UV radiation for the same time duration $t_{u v}$. The front of the colony was also observed to advance faster with respect to the higher intensity, but was still much slower compared to the $v_{f}$ before exposure to UV light (see Table 1). The total population of the colony as a function of time was measured by integrating the population densities and is shown in Fig. 3(c). For lower $I$, the population when exposed to radiation increases at a small rate initially, and then decreases. However, for higher $I$ the total population always decreases.

Qualitatively similar phenomena was observed in the two other strains of Bacillus subtilis. Although the density was observed to increase in the wild type strain near the edge of the colony, the center of the colony was not observed to vacate completely. At the same UV radiation strength, the total population and the front were also observed to grow at a faster rate compared to uvrA. In the case of uvrA recA, the colony was observed to have a higher density at the edges but the total popu- lation decreased strongly with time. Thus the behavior of the recA strain can be thought of as intermediate between that observed in the wild and the uvrA recA strains. This is consistent with the fact that while all mechanisms of DNA repair are present in the wild type, one mechanism of repair is absent in uvrA, and two are absent in the urvA recA strain [13 16]. Therefore the rate and strength of the density distribution appears to be related to the efficiency of the DNA repair mechanism.

In an attempt to elucidate the mechanism of this morphological transition, we propose the following simple model [17]. The model consists of three reaction-diffusion equations for bacteria concentration $b(\mathbf{r}, t)$, waste concentration $w(\mathbf{r}, t)$, and chemoattractant concentration $c(\mathbf{r}, t)$ :

$$
\begin{aligned}
\partial_{t} b & =f b(1-w)-\mu(1-f) b-\nabla \cdot \mathbf{J}_{c}+D_{b} \nabla^{2} b \\
\partial_{t} w & =f b(1-w)+D_{w} \nabla^{2} w \\
\partial_{t} c & =(1-f) b(1-w)+D_{c} \nabla^{2} c
\end{aligned}
$$

The first terms in the r.h.s. of Eqs.(11),(2) describe the bacterial growth and the accompanying waste production, respectively. The bacterial growth is limited by the local concentration of waste $w$ and saturates when waste concentration approaches 1 . Constant $f=1$ in the absence of the UV radiation and becomes small when UV radiation is switched on. According to Eq.(3), when $f<1$, bacteria emit chemoattractant $c$ at the rate which is proportional to the bacteria concentration and also is limited by the waste concentration. The term $-\mu(1-f) b$ in Eq.(1) is responsible for the slow destruction of bacteria by the UV radiation observed experimentally. The chemotactic flux $\mathbf{J}_{c}=\alpha b(1-b) \nabla c$ is directed towards the gradient of the concentration of chemical $c$, and it saturates at large bacterial concentration $b \rightarrow 1$ (hard core repulsion). The last terms in Eqs.(11)-(3) describe linear diffusion of the components. For simplicity, we assume that the diffusion constants of all three fields are equal and rescale them to unity.

The initial colony expansion without UV $(f=1)$ is described by the first two equations with $\mathbf{J}_{c}=0$. Asymptotically $w-b \rightarrow 0$, and the system is reduced to a single Kolmogorov-Petrovsky-Piskunov-Fisher equation 18

$$
\partial_{t} b=f b(1-b)+\nabla^{2} b
$$

which has a propagating front solution with the front speed $v_{f}=2$.

The simplest way to model the effect of UV radiation is to set $f=0$ at some time $t=t_{0}$. The growth of the bacteria is arrested, and the colony expansion stops. The subsequent dynamics of the population is determined by the interplay of diffusion and chemotaxis. In the model, the bacteria immediately begin to emit chemoattractant $c$ in the regions where waste $w$ is below the limiting level $w=1$, i.e. near the edges of the colony. A ring of $c$ is 
formed, and the exponentially decaying tails of this ring extend towards the center of the colony, thereby provoking the bacteria to concentrate near the edges of the colony.

We integrated the model (11)-(3) numerically in a square $150 \times 150$ with periodic boundary conditions starting from a small radially-symmetric non-zero perturbation of $b$ in the center for $\mu=0.02, \alpha=2$. Variations in the strength of the UV radiation can be simulated by using different small values of $f\left(t>t_{0}\right)$ instead of zero. In a typical simulation we switched $f$ from 1 to 0.1 at $t=20$, when the radius of the colony was $r \approx 21$. The resulting ring pattern and the radial density distribution as a function of time are shown in Fig. 因. As in the experiment, after the UV radiation is turned on, the population starts to assemble near the edge (cf. Fig. 3(a)). Due to slow bacterial growth, the outer front continues to expand, however, at a much slower rate. The total population size slightly grows initially at $t>t_{0}$, but later declines. All this agrees completely with observed phenomenology. A more detailed discussion of the model and its comparison with experiments will be reported elsewhere.

In summary, colonies of Bacillus subtilis are observed to show a swarming ring like pattern when exposed to UV radiation. Thus the mode in which the bacterial colonies grow after exposure to UV radiation is very different from the Fisher wave like mode in which it grows prior to exposure. Using reaction-diffusion modeling, we have shown that the migration of colony under UV radiation is related to chemotaxis which is limited by the waste that Bacillus subtilis produces.

We thank Nobou Munakata of the National Cancer Center Research Institute Tokyo, Japan for supplying the mutant strains of Bacillus subtilis. We thank T. Neicu and J. Newburg-Rinn for help with the experiments. L. T. acknowledges partial support from the U.S. DOE under grant DE-FG03-95ER14516 and DE-FG0396ER14592.

$\dagger \quad$ Corresponding author

[1] M. Matsushita and H. Fujikawa, Physica A 168, 498 (1990).

[2] E. O. Budrene and H. C. Berg, Nature 349, 630 (1991).

[3] E. Ben-Jacob, et al., Nature 368, 46 (1994).

[4] L. Tsimring, et al., Phys. Rev. Lett. 75, 1859 (1995).

[5] M. P. Brenner, L. S. Levitov, and E. O. Budrene, Biophys. J. 74, 1677 (1998).

[6] Y. Kozlovsky, et al., Phys. Rev. E 59, 7025 (1999).

[7] R. Tyson, S. R. Lubkin, and J. D. Murray, Proc. R. Soc. Lond. B 266, 299 (1999).

[8] D. R. Nelson and N. M. Shnerb, Phys. Rev. E 58, 1383 (1998).
[9] K. D. Dahmen, D. R. Nelson, and N. M. Shnerb, Jour. Math. Bio. 41, 1 (2000).

[10] T. Neicu, A. Pradhan, D. A. Larochelle, and A. Kudrolli, Phys. Rev. E 62, 1059 (2000).

[11] J. W. Wakita, et al., J. Phys. Soc. Japan 63, 1205 (1994).

[12] E. O. Budrene and H. C. Berg, Nature 376, 49 (1995).

[13] J. Jagger, Solar-UV Actions on Living Cells (Praeger Publishers, New York, 1985).

[14] N. Munakata, J. Radiat. Res. 30, 338 (1989).

[15] J. H. Hanlin, S. J. Lombardi, and R. A. Slepecky, J. Bacteriology 163, 774 (1985).

[16] B. Lewin, Genes VI (Oxford University Press, New York, 1997).

[17] The model which we propose here, is similar to the model for the aggregation bacterial patterns reported in Ref. [4]. The important difference however is that in Ref. 4, the chemoattractant emission was triggered by the waste production, whereas in the present situation, the chemoattractant emission is triggered by the UV radiation and is limited by waste. This factor prevents the chemotactic instability that would lead to bacteria clumping in multiple rings or spots.

[18] A. N. Kolmogorov, I. G. Petrovsky, and N. S. Piskunov, Bull MGU A1, 1 (1937); R. Fisher, Ann. Eugenics, 7, 335 (1937).

\begin{tabular}{cccc}
\hline $\begin{array}{c}I \\
\mathrm{~W} / \mathrm{m}^{2}\end{array}$ & $\begin{array}{c}v_{f} \text { (initial) } \\
\mu \mathrm{m} \mathrm{s}^{-1}\end{array}$ & $\begin{array}{c}v_{f}(\mathrm{UV}) \\
\mu \mathrm{m} \mathrm{s}^{-1}\end{array}$ & $\begin{array}{c}v_{g}(\mathrm{UV}) \\
\mu \mathrm{m} \mathrm{s}^{-1}\end{array}$ \\
\hline \hline 30 & 0.3 & 0.004 & $0.016-0.020$ \\
12 & 0.3 & 0.006 & $0.013-0.016$ \\
7 & 0.3 & 0.010 & $0.011-0.013$ \\
\hline
\end{tabular}

TABLE I. The front and group velocity of Bacillus subtilis uvrA as a function of intensity $I$ of the UV radiation. 
FIG. 1. (a)-(c) The images of Bacillus subtilis, uvrA as a function of time $t_{u v}$ under ultraviolet (UV) radiation. (a) $t_{u v}$ $=0 \mathrm{~h}(\mathrm{~b}) t_{u v}=25 \mathrm{~h}$ and (c) $t_{u v}=45 \mathrm{~h}$. The UV radiation of strength $I=30 \mathrm{~W} / \mathrm{m}^{2}$ is switched on after the colony was allowed to grow for time $t=50$ hours. The bacterial population is observed to migrate to the edge of the colony. The bright point at the center of the colony corresponds to the point of inoculation. (d)-(f) Recovery images of the bacterial population as a function of time $t_{r}$ after the UV radiation is switched off. The bacterial colony was allowed to recover for (d) $t_{r}=30 \mathrm{~h}(\mathrm{e}) t_{r}=40 \mathrm{~h}$ and (f) $t_{r}=55 \mathrm{~h}$. The bacterial population is observed to grow both outward as well as into the vacated inner region.

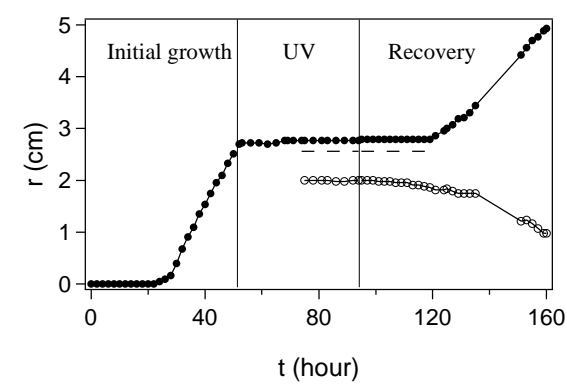

FIG. 2. The radius of the colony as a function of time from the inoculation $\left(I=30 \mathrm{~W} / \mathrm{m}^{2}\right)$. The outer edge of the colony is plotted using $(\bullet)$, and the inner edge using $(\circ)$. The bacterial colony is observed to recover both inwards and outwards in the absence of UV radiation. The dashed line corresponds to the point where population density has decreased to less than $10 \%$ of the maximum value at the edge.
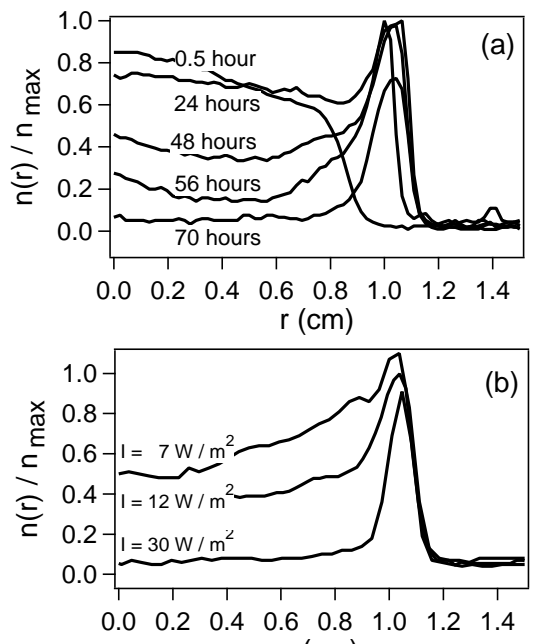
$\mathrm{r}(\mathrm{cm})$

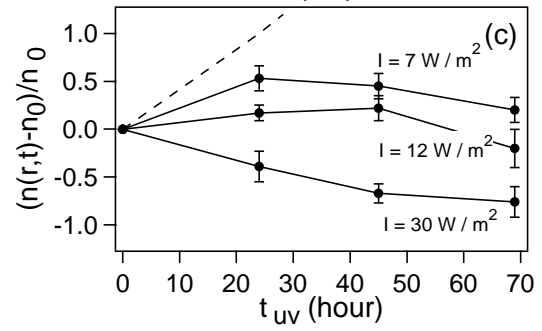

FIG. 3. (a) The population density $n(r) / n_{\max }$ as a function of distance $r$ from the inoculation point and as a function of time for $I=12 \mathrm{~W} / \mathrm{m}^{2} . n_{\max }$ is the maximum density at $t_{u v}=0$. (The data is shifted by approximately $2 \mathrm{~mm}$ from the inoculation point to avoid the puncture mark.) (b) The $n(r) / n_{\max }$ as a function of radiation strength $I$ at for 46 hours. The ring is observed to form faster as the intensity of UV radiation is increased. The colony front for the three cases have been offset to align at the colony edge for clarity. (c) The fractional change of the total population as a function of time after the UV radiation is turned on for the three intensities. The dashed line is the fractional increase in the population in the absence of UV radiation.

FIG. 4. The bacterial density $b(r)$ as a function of distance $r$ from the center at 10 unit time intervals after the radiation was effectively turned on in Eqs.(1)-(3) for $\mu=0.02, \alpha=2$. Inset: A ring formed using the proposed model is similar to that observed in the experiments. 
This figure "fig1.jpg" is available in "jpg" format from: http://arxiv.org/ps/cond-mat/0101018v2 
This figure "fig4.jpg" is available in "jpg" format from: http://arxiv.org/ps/cond-mat/0101018v2 